\title{
Heavy Atom Oriented Orbital Angular Momentum Manipulation in Metal-Free Organic Phosphors
}

\section{Wenhao Shao}

University of Michigan-Ann Arbor https://orcid.org/0000-0002-5237-0076

\section{Hanjie Jiang}

University of Michigan-Ann Arbor

\section{Paul Zimmerman}

University of Michigan-Ann Arbor https://orcid.org/0000-0002-7444-1314

Jinsang Kim ( $\boldsymbol{}_{\text {jinsang@umich.edu })}$

University of Michigan-Ann Arbor https://orcid.org/0000-0002-1235-3327

\section{Article}

Keywords: Metal-free purely organic phosphors (POPs), heavy atom effects

Posted Date: May 6th, 2021

DOl: https://doi.org/10.21203/rs.3.rs-435553/v1

License: (c) (1) This work is licensed under a Creative Commons Attribution 4.0 International License. Read Full License

Version of Record: A version of this preprint was published at Chemical Science on January 1st, 2022. See the published version at https://doi.org/10.1039/D1SC05689A. 


\title{
Heavy Atom Oriented Orbital Angular Momentum Manipulation in Metal-Free Organic Phosphors
}

\author{
Wenhao Shao $^{\dagger}$, Hanjie Jiang ${ }^{\dagger}$, Paul M. Zimmerman ${ }^{*, \dagger}$, Jinsang Kim ${ }^{*, \dagger, \star, \&, \circ}$ \\ ${ }^{\dagger}$ Department of Chemistry, ${ }^{\dagger}$ Macromolecular Science and Engineering, ${ }^{\S}$ Department of Materials \\ Science and Engineering, and ${ }^{\circ}$ Department of Chemical Engineering, University of Michigan, \\ Ann Arbor, Michigan, 48109, United States. *paulzim@umich.edu (P.M.Z.) \\ *jinsang@umich,edu (J.K.)
}

\begin{abstract}
Metal-free purely organic phosphors (POPs) are emerging materials for display technologies, solid-state lighting, and chemical sensors. The past decade has seen the promising utility of the ElSayed rule and heavy atom effects in the design of POPs, and efficient matrix engineering to boost emission efficiencies. However, due to limited contemporary design strategies, the intrinsic spinorbit coupling (SOC) efficiency of POPs remains low and their emission lifetime is pinned in the millisecond-second regime. Here, we report a universally applicable methodology to synergistically manipulate the main descriptors in SOC - heavy atom effect and orbital angular momentum, assisted by a novel set of natural-transition-orbital-based computation methods to visualize angular momentum descriptors in molecular design. Prototype POPs with efficient roomtemperature phosphorescence were designed with SOC efficiencies boosted beyond $10^{2} \mathrm{~cm}^{-1}$ and lifetime pushed below the millisecond regime. Experimental verification for our novel design rule was conducted through systematic computation-assisted design achieving discrete tuning of heavy atom effects and orbital angular momentum.
\end{abstract}


Organic phosphors are the functional components in modern technologies such as displays, solid-state lighting, and chemical sensors. While conventional organometallic phosphors suffer from metal ion dislocation ${ }^{1,2}$, device longevity problems, and toxicity issues, metal-free purely organic phosphors (POPs) have many advantageous properties such as large design windows, easy processability, economic material cost, and less toxicity ${ }^{3}$. However, POPs typically exhibit a long lifetime due to the involvement of spin-forbidden transitions, which should be facilitated by spinorbit coupling (SOC) ${ }^{4}$. To design contemporary POPs, SOC is promoted mostly by heavy atom effects and the El-Sayed rule. Fig. 1 listed a few representative POPs with halogen or chalcogen heavy atoms: $\mathrm{Br}^{5-15}, \mathrm{I}^{16,17}$, or $\mathrm{Se}^{18,19}$. The El-Sayed rule explains the necessity of $\left(\pi, \pi^{*}\right)-\left(n, \pi^{*}\right)$ transitions in promoting $\mathrm{SOC}^{20}$, and the utilization of carbonyl ${ }^{5-13}$, heterocyclic rings ${ }^{21-23}$ (e.g. triazine in $\mathrm{DPhCzT}^{21}$ ), and other moieties having rich non-bonding electrons (e.g. sulfoxide in Cs$\mathrm{Br}^{24}$ ) to stimulate low energy $\left(n, \pi^{*}\right)$ states. Due to the forbidden nature of singlet-triplet transitions and consequently slow triplet emission, matrix engineering to effectively suppress collisional quenching (the major non-radiative decay route) is essential to achieve POPs with decent quantum efficiency ${ }^{9}$. For that purpose, crystal design and strong intermolecular bonding between POPs and rigid matrix have been efficiently implemented ${ }^{5,6,9,24-32}$. The slow decay nature of POPs, on the other hand, has been explored to create persistent emitters with a long lifetime in the $10^{-1}-10^{0}$ second regime.
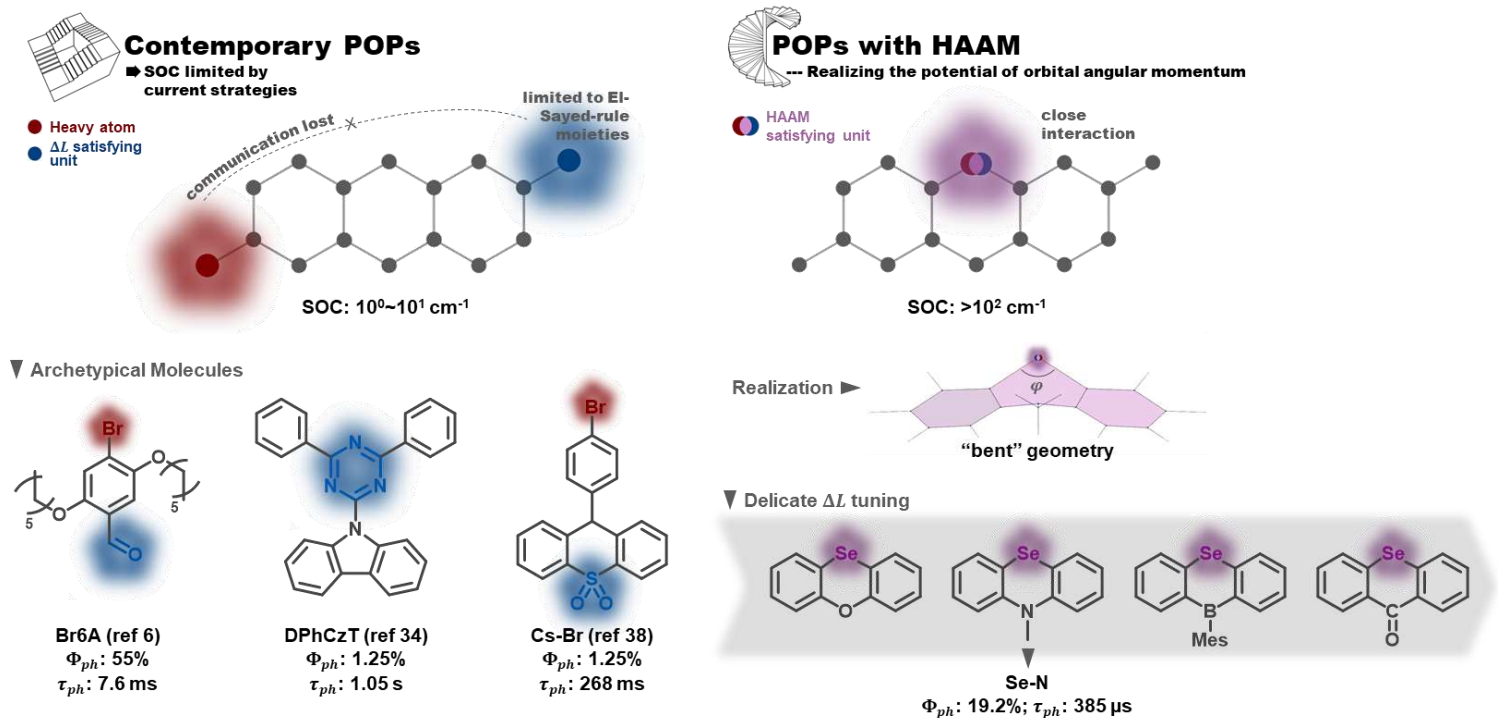

Figure 1. Contemporary POP design vs POPs designed with the heavy atom oriented angular momentum manipulation principle.

Current design strategies have reached their limit, however, and the intrinsic SOC efficiencies of POPs remain low $\left(10^{0}-10^{1} \mathrm{~cm}^{-1}\right)$ with the emission lifetime stuck in the millisecond to second regime. The key deficiency in existing strategies is the lack of synergistic interactions between the SOC-inducing components. On one hand, the El-Sayed rule isn't sufficient enough to capture the detailed picture of molecular features leading to angular momentum changes between spin states. On the other hand, the interplay between the heavy atom effect and orbital angular momentum is missing, i.e., heavy atoms are not rationally positioned in such a way that their orbitals could participate in angular momentum variation. An additional factor that has hindered creation of better design strategies is that contemporary computational tools - while able to quantify SOCs-have provided little insight into the electronic origins of the SOC within chromophores. 
Here, we report a novel methodology to synergize the heavy atom effect into close connection with orbital angular momentum and overcome the current SOC limit of organic phosphors. As demonstrated in Fig. 1, the proposed "Heavy atom oriented orbital angular momentum manipulation" (HAAM) method extends conventional El-Sayed rule to a complete interpretation of orbital angular momentum variation $(\Delta \boldsymbol{L})$ in SOC. The core of the strategy involves using heavy atoms to directly stimulate $\Delta \boldsymbol{L}$ instead of arbitrarily mixing the two elements. This strategy enhances SOCs to over $10^{2} \mathrm{~cm}^{-1}$ and pushes the lifetime limit of organic phosphors to below ms regime, as demonstrated in a newly designed series of prototype POPs using chalcogen heavy atoms. The HAAM principle is confirmed to be operational through a novel natural transition orbital (NTO)-based computational method to visualize the molecular orbital origins of SOC. In total, this work introduces the HAAM principle and tests its relevance using theory, computation, and experiment.

\section{Results}

\section{Theory behind SOC motivating the HAAM concept}

The HAAM design concept was inspired by physical principles that govern SOC. Under Fermi's golden rule, the transition rate (state 1 to state 2 ) for intersystem crossing is given as $k_{12} \propto$ $\left|\left\langle\psi_{2}\left|H_{S O}\right| \psi_{1}\right\rangle\right|^{2} \rho\left(E_{12}\right) .{ }^{33}$ Here, $\rho\left(E_{12}\right)$ denotes the joint density of states of the initial and final wavefunctions, $\psi_{1}$ and $\psi_{2} ; H_{S O}$ is the transition Hamiltonian, and the term $\left\langle\psi_{2}\left|H_{S O}\right| \psi_{1}\right\rangle$ is the SOC transition matrix element (SOCME). For one-electron systems under relativistic conditions, the main-part of the spin-orbit Hamiltonian is $\boldsymbol{H}_{S O}=\frac{Z e^{2}}{2 m^{2} c^{2} r^{3}} \boldsymbol{L S},{ }^{34}$ where $r$ is the orbital radius, $\boldsymbol{L}$ and $\boldsymbol{S}$ are the orbital and spin angular momentum, respectively. For many-electron systems, the Hamiltonian is expressed in terms of Breit-Pauli (BP) Hamiltonian ${ }^{35}$ as

$$
\boldsymbol{H}_{S O}=\alpha^{2}\left\{\sum_{n}\left[\sum_{i} \frac{Z_{n}}{r_{n i}^{3}} \boldsymbol{l}_{n i} \boldsymbol{s}_{i}-\sum_{i} \sum_{j \neq i} \frac{1}{r_{n i}^{3}} \boldsymbol{l}_{n i}\left(\boldsymbol{s}_{i}+2 \boldsymbol{s}_{j}\right)\right]\right\}
$$

where the first term represents one-electron contributions involving electrons $(i)$ and atoms $(n)$, and the second represents two-electron ( $i$ and $j$ ) interactions. Here $\alpha$ is the fine structure constant; $Z_{n}$ the atomic number of atom $n ; r_{n i}$ is the electron-nuclear distance, $\boldsymbol{l}_{n i}$ the orbital angular momentum; and $\boldsymbol{s}_{i}$ is the spin angular momentum. Since the expectation value of $r^{-3}$ is proportional to $Z^{3}$, SOCME scales with $Z^{4}$ and thus dramatically increases with heavy elements.

Intuitively, the coupling of electrons' spin and angular momentums in Eq. 1 follows the conservation of total angular momentum rule, that since $S$ changes with a spin change, the orbital angular momentum, $\boldsymbol{L}$, has to change as well. The El-Sayed rule ${ }^{20}$ qualitatively elucidated the angular momentum conservation law, for example in a typical case the $\left(\pi, \pi^{*}\right)-\left(n, \pi^{*}\right)$ transition carries a change in orbital angular momentum $(\Delta \boldsymbol{L})$, but a $\left(\pi, \pi^{*}\right)-\left(\pi, \pi^{*}\right)$ is not expected to do the same. On the other hand, large $\Delta \boldsymbol{L}$ has been observed in other types of transitions ${ }^{7,18}$, even for $\left(\pi, \pi^{*}\right)$ - $\left(\pi, \pi^{*}\right)$ transitions.

More importantly, it is clear from in Eq. 1 that orbital and spin angular momenta are coupled through the interaction with atomic number. Importantly, due to the form of Equation 1, the heavy atom must be in close proximity with the spin orbital transition, otherwise the effective $\mathrm{Z}$ from that atom is suppressed by the $\mathrm{r}^{-3}$ dependence of $H_{S O}$. While our discussion here makes these principles transparent, in practice the combination of heavy atom effects with orbital angular 
momentum change has not been systematically explored. This gives an open design space to explore the HAAM method for creating novel, tunable triplet emitters.

To help instantiate the HAAM strategy, ab initio simulations can provide insight into proposed chromophore designs by revealing their specific electronic structures and SOC elements. In particular, the restricted active space - spin flip (RAS-SF) method ${ }^{36-40}$ is a wave function theory that is well-suited for treating electronically excited states ${ }^{41,42}$ of photoactive molecular systems. RAS-SF has been shown to accurately treat a variety of challenging electronic structure problems, including polyradicals, singlet fission mechanisms, and charge transfer processes. ${ }^{43-47}$ Recent work has enabled RAS-SF to predict accurate SOC elements, making it particularly useful to complement the HAAM design concept. Furthermore and vital to instantiating the HAAM strategy, RAS-SF can produce natural transition orbitals (NTO) that couple pairs of spin states, revealing the specific changes in electronic structure that give rise to spin-orbit interactions. In this work, RAS-SF will show how the HAAM principle applies in practice to novel organic phosphors. This study therefore provides quantitative predictions of SOC and direct visualization of the interactions leading to SOC.

\section{Prototype Molecular Design and Verification of HAAM concept}

Increasing the involvement of heavy atoms in $\Delta \boldsymbol{L}$ is critical to the HAAM concept, and the best amplification should be achieved where heavy atom orbitals are involved in the electronic transition of interest. Thus, the utilization of non-bonding electrons from heavy atoms is critically important. Selenium was selected as the heavy atom due to our previous work ${ }^{18}$ that shows (unlike halogens) it can be incorporated in the core of POPs to activate $\Delta \boldsymbol{L}$ channels on its own, and additional $\Delta \boldsymbol{L}$-satisfying moieties won't be necessary. To access the non-bonding $p$ electrons from Se, we designed N-methylated phenylselenazine (Se-N, 1) carrying a bent geometry enabled by $s p^{3}$ hybridization at the nitrogen (Fig. 2a). Accordingly, $p$ electrons from Se are expected to be partially decoupled from the surrounding $\pi$-conjugated system and the non-bonding electrons could participate in $\Delta \boldsymbol{L}$.

RAS-SF calculations of Se-N show a large SOCME of $209.9 \mathrm{~cm}^{-1}$ between $\mathrm{T}_{1}$ and the ground state $\mathrm{S}_{0}$, which is the critical transition affecting phosphorescence rate. A $64.1 \mathrm{~cm}^{-1}$ SOCME for the $\mathrm{S}_{1}-\mathrm{T}_{2}$ transition was also calculated, for the critical ISC process to populate triplet excitons. A detailed analysis on the NTOs of Se-N was performed to elucidate the large SOC efficiency of Se-N and how it connects with the HAAM concept. As shown in Fig. $2 \mathrm{a}, p_{z}$ electrons of Se populated the hole orbital of $\mathrm{T}_{1}$ state while $p_{x y}$ electrons populated the electron orbital $\left(p_{x y}\right.$ is a linear combination of $p_{x}$ and $p_{y}$ ). Since orbital angular momentum rotates $90^{\circ}$ between $p_{z}$ and $p_{x y}$ orbitals, electron migration between the hole and electron orbitals of $\mathrm{T}_{1}$ would carry a large "heavy atom oriented" $\Delta \boldsymbol{L}$, enabled by the heavy Se atom. In other words, the $\mathrm{T}_{1}-\mathrm{S}_{0}$ transition of Se-N follows the HAAM concept, leading to its large SOCME. Similarly, the HAAM concept is also manifested in the $S_{1}-T_{2} S O C$ : the transition occurs through the excited electron orbitals of the $S_{1}$ and $\mathrm{T}_{2}$ states, which are populated by $p_{x y}$ and $p_{z}$ electrons of Se, respectively.

To further test the novel design principle, Se-N was synthesized and embedded in polymeric matrixes (see methods for details). In anoxic environment, fast room-temperature phosphorescence decay was observed (Fig. 2b), with measured $\tau_{p h}$ of $385 \mu$ s and $\Phi_{p h}$ of $19.2 \%$. These results are consistent with the computed SOCME of $10^{2} \mathrm{~cm}^{-1}$, which is a record-high in POPs, ${ }^{3}$ and is comparable to that of some organometallic phosphors. ${ }^{4-50}$ This large SOCME enabled by the 
HAAM concept shows that POPs can have similar emissive properties compared to their organometallic counterparts, where SOCMEs are typically in the range of $10^{2}-10^{3} \mathrm{~cm}^{-1} .{ }^{48-50}$

(a)
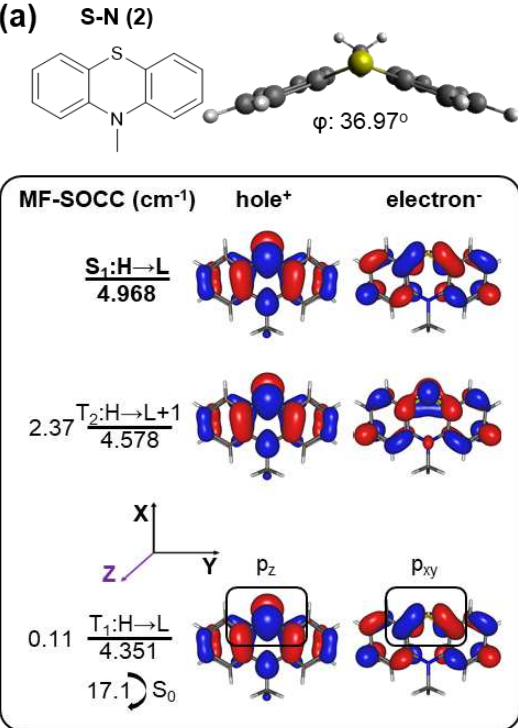

(b)
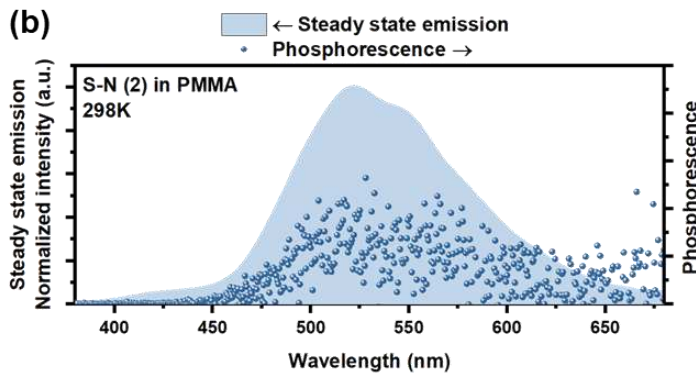
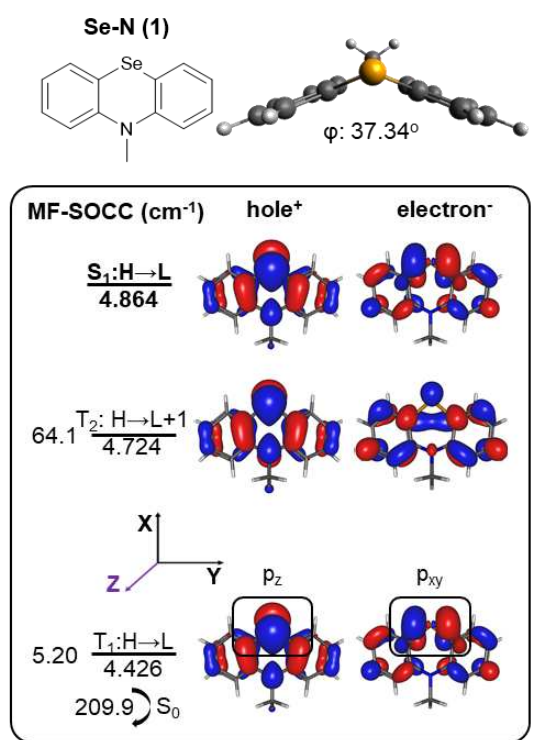

SOCME with $\mathrm{S}_{1}\left(\mathrm{~cm}^{-1}\right) \frac{\text { State }}{\text { Energy }(\mathrm{eV})}$ SOCME with $\mathrm{S}_{0}\left(\mathrm{~cm}^{-1}\right) \mathcal{S} \mathrm{S}_{0}$

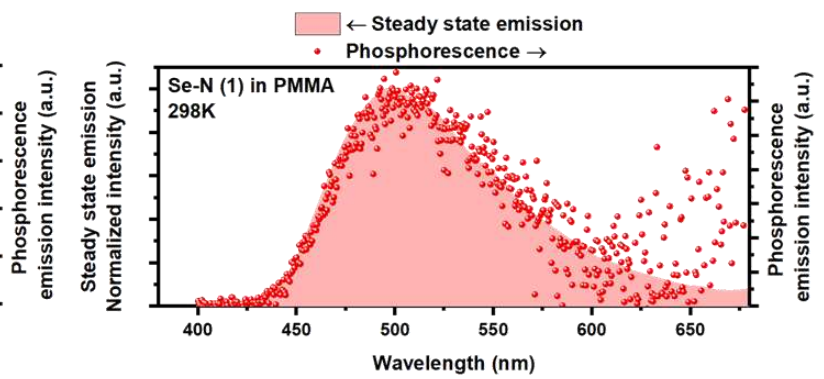

Figure 2. Computational and experimental results of Se-N (1) and Se-N (2). (a) Chemical structures of S-N and Se-N. Electronic structure results from RAS-SF showing the ground state optimized structure with bent geometry and dihedral angle and the NTOs of $S_{1}, T_{1}, T_{2}$ states. Energy of each state and the selected mean field SOCMEs are shown as well. (b) Steady state emission spectra (filled graph, left axis) and gated phosphorescence emission spectra (dot, right axis, 0.5-5 ms for Se-N and $0.5-20 \mathrm{~ms}$ for $\mathrm{S}-\mathrm{N}$ ) of the two emitters doped in atactic PMMA (1 wt\%, spin-cast) measured at room temperature in vacuum.

While the NTO analyses above provide qualitative visualization for how non-bonding $p$-electrons of Se directs the $\Delta \boldsymbol{L}$, this phenomenon could be quantified as well. Intuitively, if $\boldsymbol{\Delta} \boldsymbol{L}$ is dominated by a $p_{z}-p_{x y}$ transition, by using the right-hand rule, $\Delta \boldsymbol{L}$ should be parallel to the $x y$ plane. In other words, if we reduce the angular momentum change operator $\hat{L}$ using cartesian coordinates into $\widehat{L}_{x y}$ and $\widehat{L}_{z}$, SOC efficiency will be more pronounced with the $\hat{L}_{x y}$ operator, since the $\hat{L}_{z}$ operator only performs an orbital rotation around the $z$ axis.

RAS-SF based NTO analyses not only produce accurate representation of SOC mechanism, but also provide insights on the reduced SOCME in selected orientations, which reveal the contributions of each angular momentum operator. ${ }^{48}$ Under the same rationale, according to the matrix representation of the angular momentum in the basis of $p$-orbitals, Se $p_{z}-p_{x y}$ transition in $\mathrm{T}_{1}-\mathrm{S}_{0}$ of Se-N should produce a considerable $\left\langle p_{z}\left|\hat{L}_{x / y}\right| p_{x y}\right\rangle$ matrix element compared to that from the $\left\langle p_{z}\left|\hat{L}_{z}\right| p_{x y}\right\rangle$ operator. This is confirmed by RAS-SF results (Table 1) showing major 
contributions to SOC from the reduced components in $L_{-}$and $L_{+}$orientations ( $L_{x}$ and $L_{y}$, respectively). Most importantly, the HAAM principle is directly supported since the majority of SOC is facilitated by $\Delta \boldsymbol{L}$ on Se heavy atom. Thus, the benefit from RAS-SF methods is substantial in our discussion.

Table 1. Reduced SOCME in the selected orientations between $S_{0}$ and $T_{1}$ states

$\begin{array}{ccc}\text { Orientation } & \text { S-N } & \text { Se-N } \\ L_{x} \text { or } L_{-} & -10.50-6.02 \mathrm{i} & -137.56+0.01 \mathrm{i} \\ L_{z} \text { or } L_{0} & 0.00 & -78.77 \mathrm{i} \\ L_{y} \text { or } L_{+} & -10.50+6.02 \mathrm{i} & -137.56-0.01 \mathrm{i}\end{array}$

The HAAM concept was further examined by discretely tuning the heavy atom effect and $\Delta \boldsymbol{L}$. First, heavy atom effect was measured by replacing Se in Se-N with S while keeping $\Delta \boldsymbol{L}$ relatively consistent. Frontier excited states of the designed molecule, S-N, have similar NTOs to those of Se-N, suggesting similar $\Delta \boldsymbol{L}$ is present in the relevant electronic transitions. As expected, the SOCMEs of S-N were much smaller than those of the Se counterpart (e.g., 17.1 vs $209.9 \mathrm{~cm}^{-1} \mathrm{~T}_{1^{-}}$ $\mathrm{S}_{0} \mathrm{SOCME}$ ). Table 1 also indicates largely reduced SOCME of S-N for the reduced components in all three orientations.

Secondly, Se was reintroduced and the contribution of heavy atom orbitals in $\Delta \boldsymbol{L}$ was examined over a range of angular momentum changes. This was achieved via simulation by measuring the $\mathrm{SOC}$ for $\mathrm{T}_{1}-\mathrm{S}_{0}$ along various bending angles (Fig. 3a). The contribution of Se $p_{x y}$ orbitals in the $\mathrm{T}_{1}$ state gradually increased as the bending angle was enlarged from 10 to $60^{\circ}$ since $p_{x y}$ electrons are gradually decoupled from the $\pi$-conjugated system, leading to the increased $\Delta \boldsymbol{L}$ and $\mathrm{T}_{1}-\mathrm{S}_{0}$ SOCME (Fig. 3b). Similar trends also exist in the reduced SOCMEs in $L_{-}, L_{0}$, and $L_{+}$orientations (Fig. S1, Table S2).

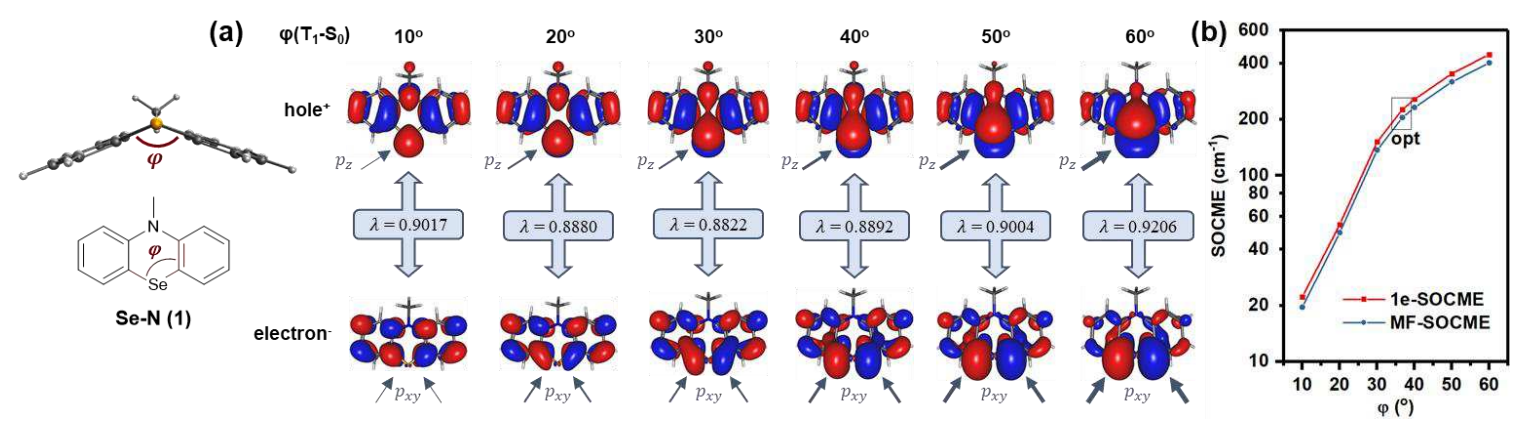

Figure 3. Computational results of Se-N (1) scanned through the dihedral angle. (a) $\mathrm{T}_{1}-\mathrm{S}_{0} \mathrm{NTO}$ of Se-N optimized with dihedral angle $(\varphi)$ fixed; $\lambda$ represents the contribution of the NTO pair in SOC (max. 1). (b) RAS-SF one-electron and mean-field SOCMEs of $\mathrm{T}_{1}-\mathrm{S}_{0}$ transition v.s. dihedral angle, $\varphi\left({ }^{\circ}\right)$.

To probe the dihedral degree of freedom via experiment, the functional group opposite to Se provides a possible handle. Thus, a series of molecules was designed where the nitrogen in Se-N is replaced by oxygen, boron, or carbonyl (Fig. 4a). With these substitutions, the dihedral angle was reduced from $\sim 37^{\circ}$ to $0^{\circ}$ (Fig. $4 \mathrm{~d}$ ) due to a change in orbital hybridization from $s p^{3}(\mathrm{~N})$ to $s p^{2}(\mathrm{CO})$. Accordingly, $\Delta \boldsymbol{L}$ is expected to decrease since non-bonding electrons of Se experience a higher degree of conjugation with the nearby ring system. Since Se is a much heavier atom than oxygen, boron, or carbonyl, the heavy atom effect should remain approximately constant. In line with expectations, the calculated $\mathrm{T}_{1}-\mathrm{S}_{0}$ SOCMEs show a decreasing trend upon reducing the bending angle from Se-N through Se-B to Se-CO (Fig. 4b,d). A similar trend is observed in the primary 
SOCME component in the $L_{ \pm}$direction (Fig. 4c, Table S1). Besides the bending angle, which affects the rotation of $\boldsymbol{L}$, the induction effect of the substituents controls the electron density on $\mathrm{Se}$ and consequently the absolute value of $\boldsymbol{L}$. This could explain the larger $\mathrm{T}_{1}-\mathrm{S}_{0} \mathrm{SOCME}$ value of $\mathrm{Se}-\mathrm{O}$ than that of Se-N, despite its smaller dihedral angle.

(a)

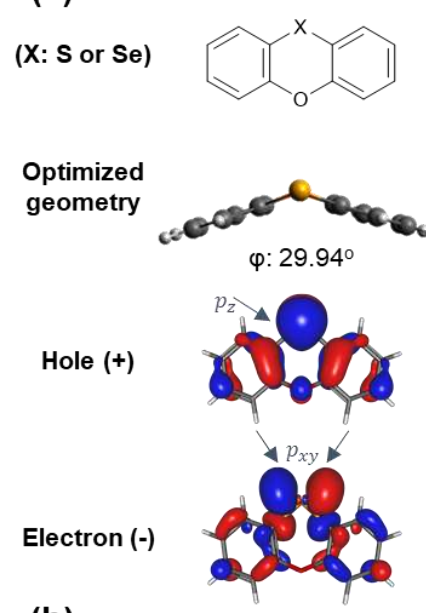

(b)
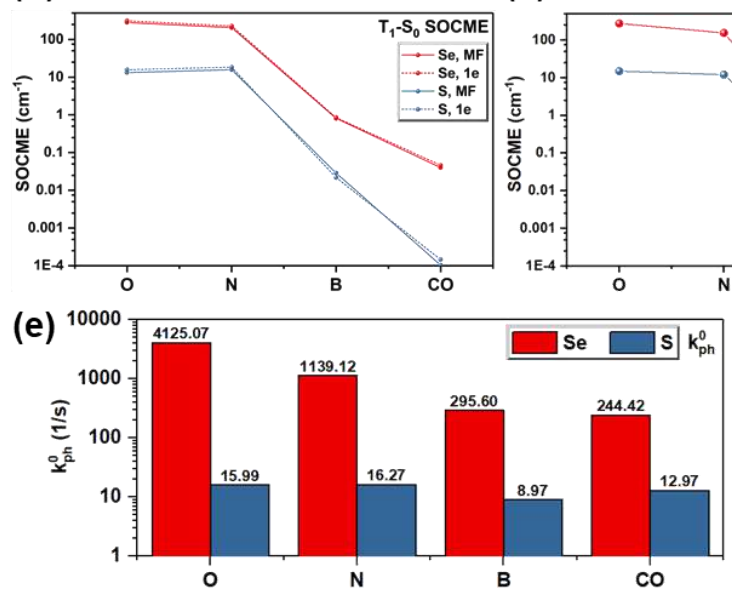

S-N (1) \& Se-N (2)
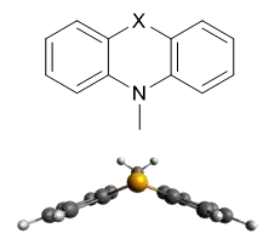

$\varphi: 37.34^{\circ}$
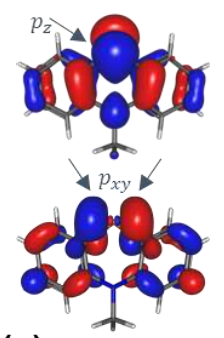

(c)

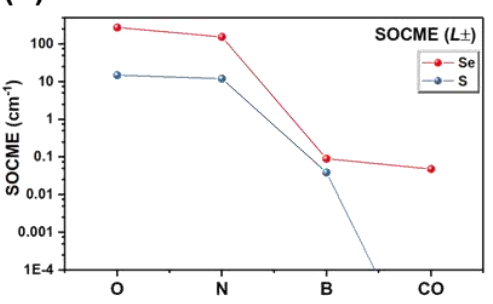

S-B (5) \& Se-B (6)
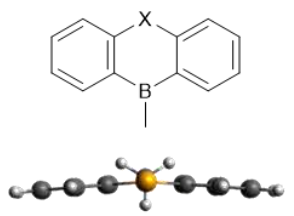

$\varphi: 8.13^{\circ}$
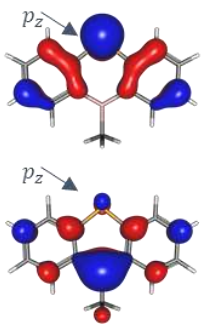

(d)
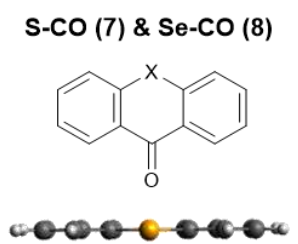

$\varphi: 0.04^{\circ}$
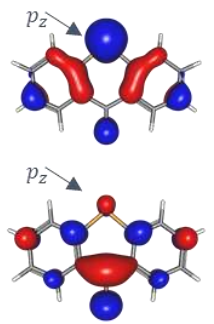

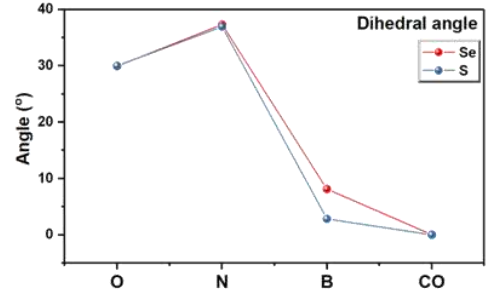

(f)

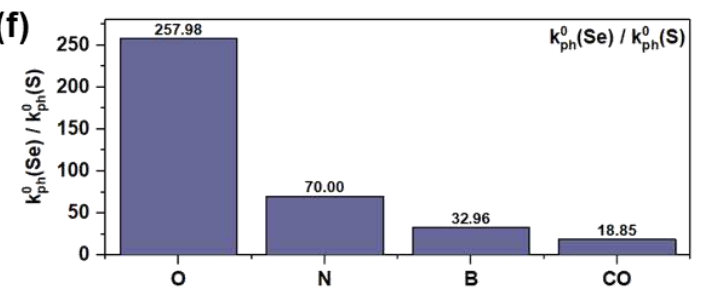

Figure 4. Computational and experimental results for $\mathrm{S} / \mathrm{Se}-\mathrm{O}, \mathrm{S} / \mathrm{Se}-\mathrm{N}, \mathrm{S} / \mathrm{Se}-\mathrm{B}$, and $\mathrm{S} / \mathrm{Se}-\mathrm{CO}$. (a) Molecular structures of the oxygen, nitrogen, boron, and carbonyl derivatives ( $X=S$ or $S e)$, their optimized ground state geometry with dihedral angle marked, and RAS-SF NTOs of $\mathrm{T}_{1}$ states with Se $p_{z}$ and $p_{x y}$ orbitals marked; (b) RAS SF one-electron (1e) and mean-field (MF) SOCMEs of $\mathrm{T}_{1}-\mathrm{S}_{0}$ transition, (c) the reduced 1e SOCME in $L_{-}$or $L_{+}$orientations (modulus) v.s. functional groups; (d) the dihedral angles of molecules studied v.s. their functional groups; (e) the experimental intrinsic phosphorescence rate $k_{p h}^{0}$ measured in doped PMMA matrix at $78 \mathrm{~K}$ in vacuum, and (f) the $k_{p h}^{0}(\mathrm{Se}$ derivative $) / k_{p h}^{0}$ (S derivative) value of each functional group.

The abovementioned computational results along with the molecular design rationale were experimentally checked by analyzing the intrinsic phosphorescence rate $\left(k_{p h}^{0}\right)$ measured at $78 \mathrm{~K}$. As expected, $k_{p h}^{0}$ decreased dramatically as the dihedral flattens in the series from Se-O to Se-CO. However, this observation alone only indirectly supports that the dihedral angle, or $\Delta \boldsymbol{L}$ variation, leads to $k_{p h}^{0}$ variation. To gain further insight, $k_{p h}^{0}$ of each Se compound is compared with its $\mathrm{S}$ counterpart (e.g. Se-N v.s. S-N). While all four derivatives showed remarkable enhancement in $k_{p h}^{0}$ by replacing $\mathrm{S}$ with $\mathrm{Se}$ in the same molecular frame (Fig. 4e), the degree of $k_{p h}^{0}$ boost, characterized 
by $k_{p h}^{0}$ (Se-derivative) $/ k_{p h}^{0}$ (S-derivative), is different with each molecular frame. It is worth

addressing that the degree of $k_{p h}^{0}$ boost follows the dihedral angle, since a larger $\Delta \boldsymbol{L}$ would enhance the contribution of heavy atom orbitals, and thus lead to more prominent heavy atom effects. Experimental results suggested the same trend (Fig. 4f) in the degree of $k_{p h}^{0}$ boost, which increased from Se-CO (18.85), through Se-B, Se-N, to Se-O (a remarkable 258-fold), strongly implying the beneficial effect of the increased dihedral angle and $\mathrm{T}_{1}-\mathrm{S}_{0} \Delta \boldsymbol{L}$. These experimental results therefore demonstrate that the "heavy atom oriented" $\Delta \boldsymbol{L}$ can be effective in practice, as motivated and expected by the HAAM concept.

\section{Discussion}

The above analysis introduces the "Heavy Atom Oriented Orbital Angular Momentum Manipulation" (HAAM) as a novel design principle for POPs and tested the concept using simulations and experiments. This HAAM concept enables a powerful use of SOC theory in molecular design, and shows potential to give control over SOC in triplet emitters. Highly efficient POPs with fast emission were realized with this strategy, providing prototype POPs with $k_{p h}^{0}$ over $10^{3} \mathrm{~cm}^{-1}$ and promising room-temperautre phosphorescence (RTP) properties (i.e. Se-N and derivatives).

This advance was supported by a novel computational method, RAS-SF, which can accurately quantify SOC in triplet emitters. RAS-SF also provides detailed NTO analysis to provide direct computational supports for the HAAM concept by revealing the electronic changes from triplet to singlet state that come with SOC. Importantly, the connection between experimental findings and systematic RAS-SF-assisted molecular design gave strong evidence that the tuning of heavy atom effects and orbital angular momentum - in synergy - is vital to increasing SOC in organic emitters. In other words, the combined quantitative and qualitative aspects of RAS-SF allowed the HAAM concept to be successfully translated from theory to practice for the design of POPs.

This combination of HAAM principle with the RAS-SF SOC method can be extended to other organic triplet-based emitter scaffolds. Whereas the HAAM concept is enacted in practice to Se-N in this work, the key to efficient HAAM-based POPs is activating the contribution of heavy atom orbitals in $\boldsymbol{\Delta} \boldsymbol{L}$, and the key to activate efficient $\Delta \boldsymbol{L}$ channels is utilizing non-bonding electrons of heavy atoms. The "bent geometry" as investigated here is expected to be just one efficient molecular scaffold to fulfill this task. A related, but less powerful method is to place heavy atoms adjacent to conventional El-Sayed rule satisfying moieties, so that they could participate in the $\left(\pi, \pi^{*}\right)-\left(n, \pi^{*}\right)$ type $\Delta \boldsymbol{L}$. This design rationale employs halogen-containing POPs,${ }^{7,8}$ since halogens only has one available bonding site and could not create efficient $\Delta \boldsymbol{L}$ channels on their own. While the fused-ring motif of the present article appears to efficiently utilize the HAAM concept by connecting the heavy atom within the $\Delta \boldsymbol{L}$-producing channel, heavy chalcogen atoms could also be placed in attachable pendent groups to create $\Delta \boldsymbol{L}$ channels, such as coupling them with ester or anhydride groups, or directly replace the oxygen in carbonyl with heavier chalcogens. However, these moieties haven't been systematically explored yet.

Further exploration of the HAAM principle will focus on expanding the library of HAAMbased POPs, in order to further break the SOC limits of POPs. Another promising direction is to further break the upper SOC limit for POPs using HAAM principle, and create new OLEDs that outperform their metal-organic counterparts. 


\section{Methods}

General. All chemicals used were purchased from Millipore Sigma or Fisher Scientific unless specified and used without further purification. (2-Bromophenyl)hydrazine was purchased from Oakwood Products, Inc.. Deuterated solvents for NMR spectroscopy (nuclear magnetic resonance) were purchased from Cambridge Isotope Laboratories. Phenoxathiine $(\mathrm{S}-\mathrm{O}, 98.0+\%)$ and $9 \mathrm{H}-$ thioxanthen-9-one (S-CO, 98.0+\%) were purchased from TCI America and used without further purification. 9H-selenoxanthen-9-one (Se-CO) was purchased form Millipore Sigma and used without purification.

Physical measurements. Nuclear Magnetic Resonance (NMR) spectra were collected on Varian MR400 (400 MHz), Varian Vnmrs 500 (500 MHz), or Varian Vnmrs 700 (700 MHz) spectrometer as indicated. Photoluminescence spectra were collected on a Photon Technologies International (PTI) QuantaMaster spectrofluorometer (QM-400) equipped with an integrating sphere (K-Sphere) and a cryostat. The emitters were doped in atactic PMMA matrix for solid-state measurements: quartz substrates $(1.5 * 2.5 \mathrm{~cm})$ were prepared and cleaned by sonication consecutively in soap, deionized water, acetone, isopropyl alcohol, and then proceeded to UV-ozone treatment for $30 \mathrm{~min}$. Chloroform solution containing $0.025 \mathrm{wt} \%$ emitter and $2.5 \mathrm{wt} \%$ PMMA was prepared and spincoated on the cleaned quartz substrates $(500 \mathrm{rpm}$ for $5 \mathrm{~min}$ ). Last, the films were transferred into a glovebox filled with $\mathrm{N}_{2}$ and baked at $120{ }^{\circ} \mathrm{C}$ for $30 \mathrm{~min}$.

\section{Acknowledgements}

The authors thank Ramin Ansari for the synthesis of precursor of Se-N. We acknowledged the financial support from National Science Foundation (DMREF DMR 1435965) and Samsung Global Research Outreach.

\section{Author contributions}

W.S. synthesized the materials and conducted the photophysical analyses, and wrote the manuscript; H. J. performed all computation analyses and contributed to the computation sections of the article; J.K. and P.M.Z. supervised the research and revised this article.

\section{Additional Information}

The authors declare no competing financial interests. The data that support the findings of this study are available from the corresponding author upon request.

\section{References}

1. Schmidbauer, S., Hohenleutner, A. \& König, B. Chemical Degradation in Organic Lightemitting Devices: Mechanisms and Implications for the Design of New Materials. Advanced Materials 25, 2114-2129 (2013).

2. Jeong, C. et al. Understanding Molecular Fragmentation in Blue Phosphorescent Organic Light-emitting Devices. Organic Electronics 64, 15-21 (2019). 
3. Mukherjee, S. \& Thilagar, P. Recent Advances in Purely Organic Phosphorescent Materials. Chemical Communications 51, 10988-11003 (2015).

4. Turro, N. J., Ramamurthy, V. \& Scaiano, J. C. Modern Molecular Photochemistry of Organic Molecules. (University Science Books, 2017).

5. Bolton, O., Lee, K., Kim, H. J., Lin, K. Y. \& Kim, J. Activating Efficient Phosphorescence From Purely Organic Materials by Crystal Design. Nature Chemistry 3, 205-210 (2011).

6. Lee, D. et al. Room Temperature Phosphorescence of Metal-free Organic Materials in Amorphous Polymer Matrices. Journal of the American Chemical Society 135, 6325-6329 (2013).

7. Song, B., Shao, W., Jung, J., Yoon, S. J. \& Kim, J. Organic Light-Emitting Diode Employing Metal-Free Organic Phosphor. ACS Applied Materials and Interfaces 12, 6137-6143 (2020).

8. Sarkar, S. et al. Phosphorescence in Bromobenzaldehyde Can Be Enhanced through Intramolecular Heavy Atom Effect. Journal of Physical Chemistry C 121, 3771-3777 (2017).

9. Kwon, M. S. et al. Suppressing Molecular Motions for Enhanced Room Temperature Phosphorescence of Metal-free Organic Materials. Nature Communications 6, (2015).

10. Zang, L., Shao, W., Kwon, M. S., Zhang, Z. \& Kim, J. Photoresponsive Luminescence Switching of Metal-Free Organic Phosphors Doped Polymer Matrices. Advanced Optical Materials 8, (2020).

11. Xu, J., Takai, A., Kobayashi, Y. \& Takeuchi, M. Phosphorescence From a Pure Organic Fluorene Derivative in Solution at Room Temperature. Chemical Communications 49, 8447-8449 (2013).

12. Gong, Y. et al. Achieving Persistent Room Temperature Phosphorescence and Remarkable Mechanochromism from Pure Organic Luminogens. Advanced Materials 27, 6195-6201 (2015).

13. Chen, X. et al. Versatile Room-Temperature-Phosphorescent Materials Prepared from NSubstituted Naphthalimides: Emission Enhancement and Chemical Conjugation. Angewandte Chemie - International Edition 55, 9872-9876 (2016).

14. She, P. et al. Controlling Organic Room Temperature Phosphorescence through External Heavy-Atom Effect for White Light Emission and Luminescence Printing. Advanced Optical Materials 8, 1-7 (2020).

15. Ma, J. L. et al. Metal-Free Room-Temperature Phosphorescence from Amorphous Triarylborane-Based Biphenyl. Organometallics 39, 4153-4158 (2020).

16. Gutierrez, G. D., Sazama, G. T., Wu, T., Baldo, M. A. \& Swager, T. M. Red Phosphorescence from Benzo[2,1,3]thiadiazoles at Room Temperature. Journal of Organic Chemistry 81, 4789-4796 (2016). 
17. Mao, Z. et al. Linearly Tunable Emission Colors Obtained from a FluorescentPhosphorescent Dual-Emission Compound by Mechanical Stimuli. Angewandte Chemie International Edition 54, 6270-6273 (2015).

18. Lee, D. R. et al. Heavy Atom Effect of Selenium for Metal-Free Phosphorescent LightEmitting Diodes. Chemistry of Materials 32, 2583-2592 (2020).

19. Kremer, A. et al. Walking Down the Chalcogenic Group of the Periodic Table: From Singlet to Triplet Organic Emitters. Chemistry - A European Journal 21, 15377-15387 (2015).

20. El-Sayed, M. A. Spin-orbit Coupling and the Radiationless Processes in Nitrogen Heterocyclics. The Journal of Chemical Physics 38, 2834-2838 (1963).

21. An, Z. et al. Stabilizing Triplet Excited States for Ultralong Organic Phosphorescence. Nature Materials 14, 685-690 (2015).

22. Lv, A. et al. Room-Temperature Phosphorescence from Metal-Free Organic Materials in Solution: Origin and Molecular Design. Journal of Physical Chemistry Letters 10, 10371042 (2019).

23. Chen, C. et al. Carbazole Isomers Induce Ultralong Organic Phosphorescence. Nature Materials 20, 175-180 (2021).

24. Yang, J. et al. The Influence of the Molecular Packing on the Room Temperature Phosphorescence of Purely Organic Luminogens. Nature Communications 9, 1-10 (2018).

25. Bolton, O., Lee, D., Jung, J. \& Kim, J. Tuning the Photophysical Properties of Metal-free Room Temperature Organic Phosphors via Compositional Variations in Bromobenzaldehyde/dibromobenzene Mixed Crystals. Chemistry of Materials 26, 6644 6649 (2014).

26. Kwon, M. S., Lee, D., Seo, S., Jung, J. \& Kim, J. Tailoring Intermolecular Interactions for Efficient Room-Temperature Phosphorescence from Purely Organic Materials in Amorphous Polymer Matrices. Angewandte Chemie 126, 11359-11363 (2014).

27. Yang, X. \& Yan, D. Long-Afterglow Metal-Organic Frameworks: Reversible GuestInduced Phosphorescence Tunability. Chemical Science 7, 4519-4526 (2016).

28. Zhou, B. \& Yan, D. Hydrogen-Bonded Two-Component Ionic Crystals Showing Enhanced Long-Lived Room-Temperature Phosphorescence via TADF-Assisted Förster Resonance Energy Transfer. Advanced Functional Materials 29, (2019).

29. Cheng, Z. et al. Ultralong Phosphorescence from Organic Ionic Crystals under Ambient Conditions. Angewandte Chemie - International Edition 57, 678-682 (2018).

30. Hamzehpoor, E. \& Perepichka, D. F. Crystal Engineering of Room Temperature Phosphorescence in Organic Solids. Angewandte Chemie - International Edition 59, 9977-9981 (2020).

31. Zhang, Z. Y., Chen, Y. \& Liu, Y. Efficient Room-Temperature Phosphorescence of a Solid-State Supramolecule Enhanced by Cucurbit[6]uril. Angewandte Chemie International Edition 58, 6028-6032 (2019). 
32. Zhang, Z. Y. \& Liu, Y. Ultralong Room-temperature Phosphorescence of a Solid-state Supramolecule Between Phenylmethylpyridinium and Cucurbit[6]uril. Chemical Science 10, 7773-7778 (2019).

33. Forrest, S. R. Organic Electronics: Foundations to Applications. (Oxford Scholarship Online, 2020). doi:10.1093/oso/9780198529729.001.0001.

34. McGlynn, S. P., Azumi, T. \& Kinoshita, M. Molecular Spectroscopy of the Triplet State. (Prentice-Hall, 1969).

35. Yarkony, D. R. Spin-forbidden Chemistry within the Breit-pauli Approximation. International Reviews in Physical Chemistry 11, 195-242 (1992).

36. Chien, A. D. \& Zimmerman, P. M. Recovering Dynamic Correlation in Spin Flip Configuration Interaction Through a Difference Dedicated Approach. Journal of Chemical Physics 146, (2017).

37. Bell, F., Zimmerman, P. M., Casanova, D., Goldey, M. \& Head-Gordon, M. Restricted Active Space Spin-flip (RAS-SF) with Arbitrary Number of Spin-flips. Physical Chemistry Chemical Physics 15, 358-366 (2013).

38. Zimmerman, P. M., Bell, F., Goldey, M., Bell, A. T. \& Head-Gordon, M. Restricted Active Space Spin-flip Configuration Interaction: Theory and Examples for Multiple Spin Flips With Odd Numbers of Electrons. Journal of Chemical Physics 137, (2012).

39. Jiang, H. \& Zimmerman, P. M. Charge Transfer via Spin Flip Configuration Interaction: Benchmarks and Application to Singlet Fission. Journal of Chemical Physics 153, (2020).

40. Casanova, D. \& Head-Gordon, M. Restricted Active Space Spin-Flip Configuration Interaction Approach: Theory, Implementation and Examples. Physical Chemistry Chemical Physics 11, 9779-9790 (2009).

41. Krylov, A. I. Spin-Flip Configuration Interaction: An Electronic Structure Model That Is Both Variational and Size-Consistent. Chemical Physics Letters 350, 522-530 (2001).

42. Krylov, A. I. Size-Consistent Wave Functions for Bond-Breaking: The Equation-ofMotion Spin-Flip Model. Chemical Physics Letters 338, 375-384 (2001).

43. Kim, H. et al. Enacting Two-Electron Transfer from a Double-Triplet State of Intramolecular Singlet Fission. Journal of the American Chemical Society 140, 7760-7763 (2018).

44. Kim, H. \& Zimmerman, P. M. Coupled Double Triplet State in Singlet Fission. Physical Chemistry Chemical Physics vol. 20 30083-30094 (2018).

45. Zimmerman, P. M., Bell, F., Casanova, D. \& Head-Gordon, M. Mechanism for Singlet Fission in Pentacene and Tetracene: From Single Exciton to Two Triplets. Journal of the American Chemical Society 133, 19944-19952 (2011).

46. Chien, A. D. et al. Structure and Dynamics of the 1(TT) State in a Quinoidal Bithiophene: Characterizing a Promising Intramolecular Singlet Fission Candidate. Journal of Physical Chemistry C 119, 28258-28268 (2015). 
47. Rudenko, A. E. et al. Ligand-Induced Reductive Elimination of Ethane from Azopyridine Palladium Dimethyl Complexes. Journal of the American Chemical Society 140, 1140811415 (2018).

48. Pokhilko, P. \& Krylov, A. I. Quantitative El-Sayed Rules for Many-Body Wave Functions from Spinless Transition Density Matrices. Journal of Physical Chemistry Letters 10, 4857-4862 (2019).

49. Xiao, L. et al. Recent Progresses on Materials for Electrophosphorescent Organic LightEmitting Devices. Advanced Materials vol. 23 926-952 (2011).

50. Mori, K., Goumans, T. P. M., van Lenthe, E. \& Wang, F. Predicting Phosphorescent Lifetimes and Zero-Field Splitting of Organometallic Complexes With Time-Dependent Density Functional Theory Including Spin-Orbit Coupling †. Phys. Chem. Chem. Phys 16, 14523 (2014).

\section{Figure Legends and Tables}

Figure 1. Contemporary POP design vs POPs designed with the heavy atom oriented angular momentum manipulation principle.

Figure 2. Computational and experimental results of Se-N (1) and Se-N (2). (a) Chemical structures of S-N and Se-N. Electronic structure results from RAS-SF showing the ground state optimized structure with bent geometry and dihedral angle and the NTOs of $S_{1}, T_{1}, T_{2}$ states. Energy of each state and the selected mean field SOCMEs are shown as well. (b) Steady state emission spectra (filled graph, left axis) and gated phosphorescence emission spectra (dot, right axis, 0.5-5 ms for Se-N and $0.5-20 \mathrm{~ms}$ for S-N) of the two emitters doped in atactic PMMA (1 wt\%, spin-cast) measured at room temperature in vacuum.

Figure 3. Computational results of Se-N (1) scanned through the dihedral angle. (a) $\mathrm{T}_{1}-\mathrm{S}_{0} \mathrm{NTOs}$ of Se-N optimized with dihedral angle $(\varphi)$ fixed; $\lambda$ represents the contribution of the NTO pair in SOC (max. 1). (b) RAS-SF one-electron and mean-field SOCMEs of $\mathrm{T}_{1}-\mathrm{S}_{0}$ transition v.s. dihedral angle, $\varphi\left({ }^{\circ}\right)$.

Figure 4. Computational and experimental results for $\mathrm{S} / \mathrm{Se}-\mathrm{O}, \mathrm{S} / \mathrm{Se}-\mathrm{N}, \mathrm{S} / \mathrm{Se}-\mathrm{B}$, and $\mathrm{S} / \mathrm{Se}-\mathrm{CO}$. (a) Molecular structures of the oxygen, nitrogen, boron, and carbonyl derivatives ( $X=S$ or $S e)$, their optimized ground state geometry with dihedral angle marked, and RAS-SF NTOs of $\mathrm{T}_{1}$ states with Se $p_{z}$ and $p_{x y}$ orbitals marked; (b) RAS SF one-electron (1e) and mean-field (MF) SOCMEs of $\mathrm{T}_{1}-\mathrm{S}_{0}$ transition, (c) the reduced 1e SOCME in $L_{-}$or $L_{+}$orientations (modulus) v.s. functional groups; (d) the dihedral angles of molecules studied v.s. their functional groups; (e) the experimental intrinsic phosphorescence rate $k_{p h}^{0}$ measured in doped PMMA matrix at $78 \mathrm{~K}$ in vacuum, and (f) the $k_{p h}^{0}($ Se derivative $) / k_{p h}^{0}$ (S derivative) value of each functional group.

Table 1. Reduced SOCME in the selected orientations between $S_{0}$ and $T_{1}$ states

$\begin{array}{ccc}\text { Orientation } & \text { S-N } & \text { Se-N } \\ L_{x} \text { or } L_{-} & -10.50-6.02 \mathrm{i} & -137.56+0.01 \mathrm{i} \\ L_{z} \text { or } L_{0} & 0.00 & -78.77 \mathrm{i} \\ L_{y} \text { or } L_{+} & -10.50+6.02 \mathrm{i} & -137.56-0.01 \mathrm{i}\end{array}$


Figures

\section{Contemporary POPs}

\section{$\Rightarrow$ soc limited by}

current strategies limited to EI-

Heavy atom

unit $\Delta L$ atisfying

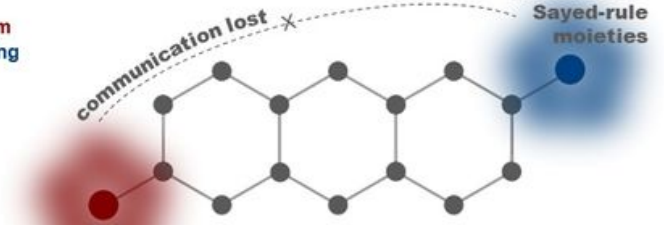

SOC: $10^{0} \sim 10^{1} \mathrm{~cm}^{-1}$

$\nabla$ Archetypical Molecules

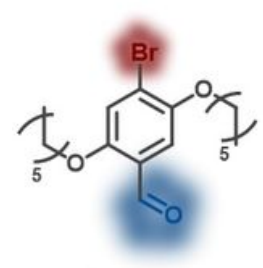

Br6A (ref 6)

$\Phi_{p h}: 55 \%$

$\tau_{p h}: 7.6 \mathrm{~ms}$

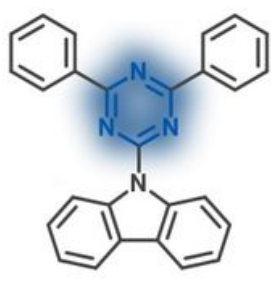

DPhCzT (ref 34)

$\Phi_{p h}: 1.25 \%$

$\tau_{p h}: 1.05 \mathrm{~s}$

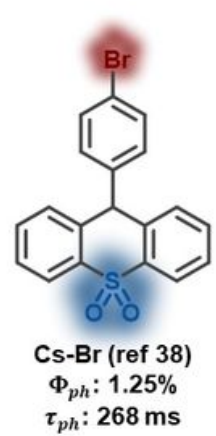

POPs with HAAM

-.- Realizing the potential of orbital angular momentum

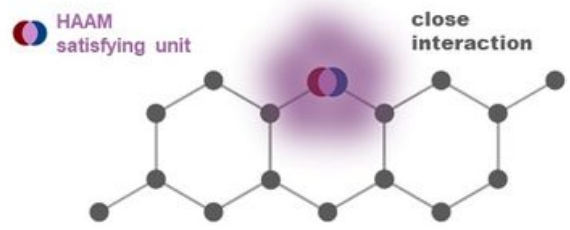

SOC: $>10^{2} \mathrm{~cm}^{-1}$

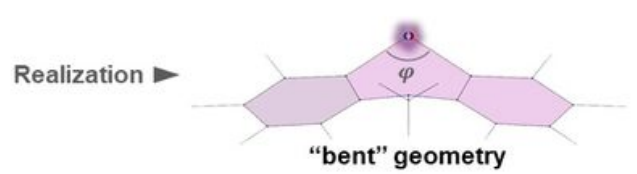

$\nabla$ Delicate $\Delta L$ tuning

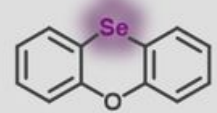

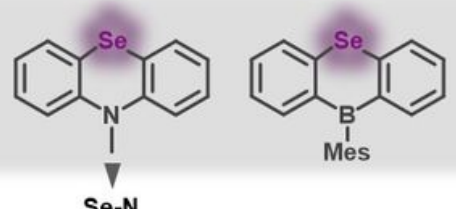

Se-N

$\Phi_{p h}: 19.2 \% ; \tau_{p h}: 385 \mu \mathrm{s}$

\section{Figure 1}

Contemporary POP design vs POPs designed with the heavy atom oriented angular momentum manipulation principle. 
(a)
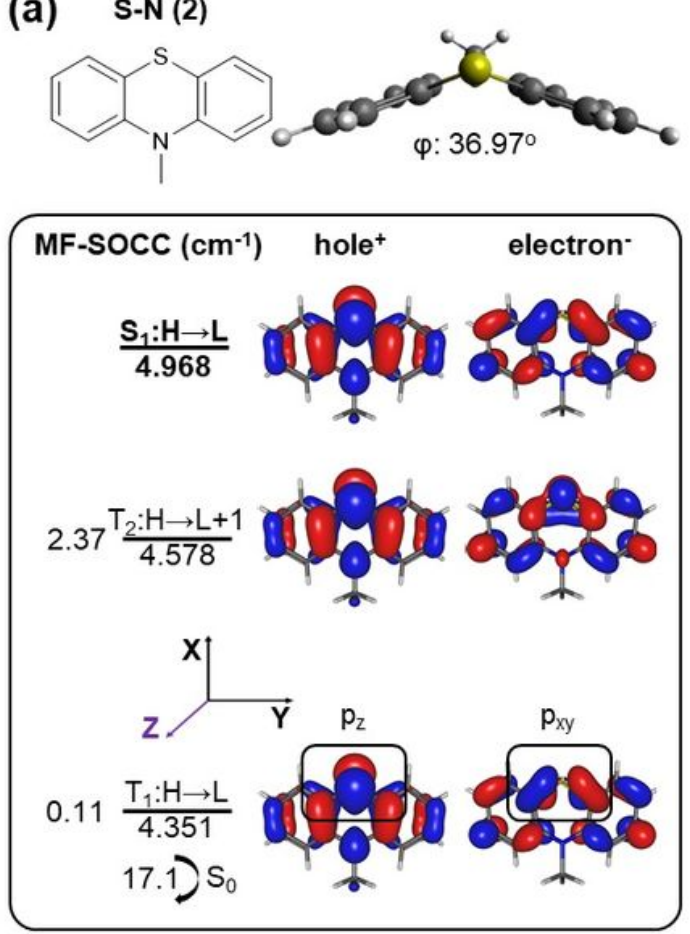

(b)

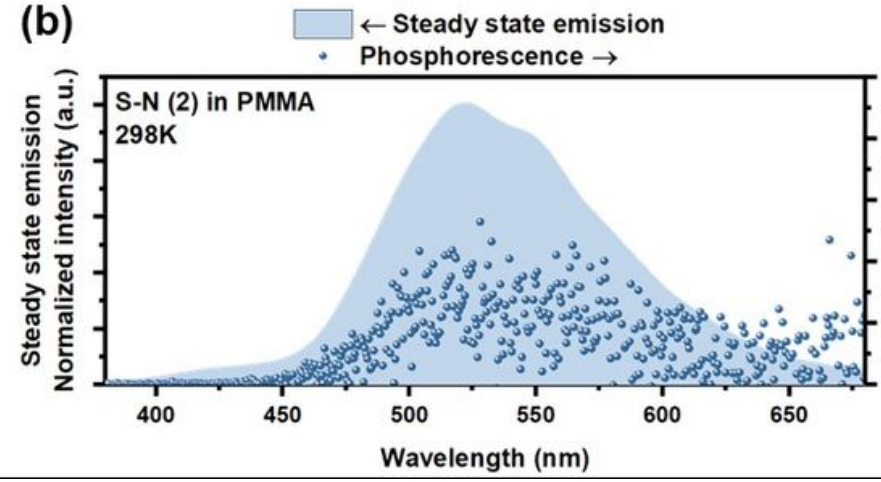

Se-N (1)

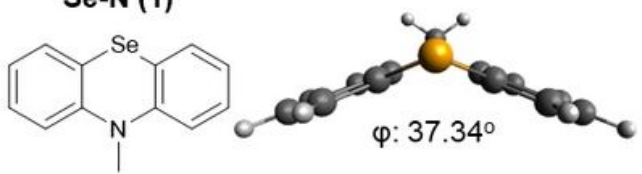

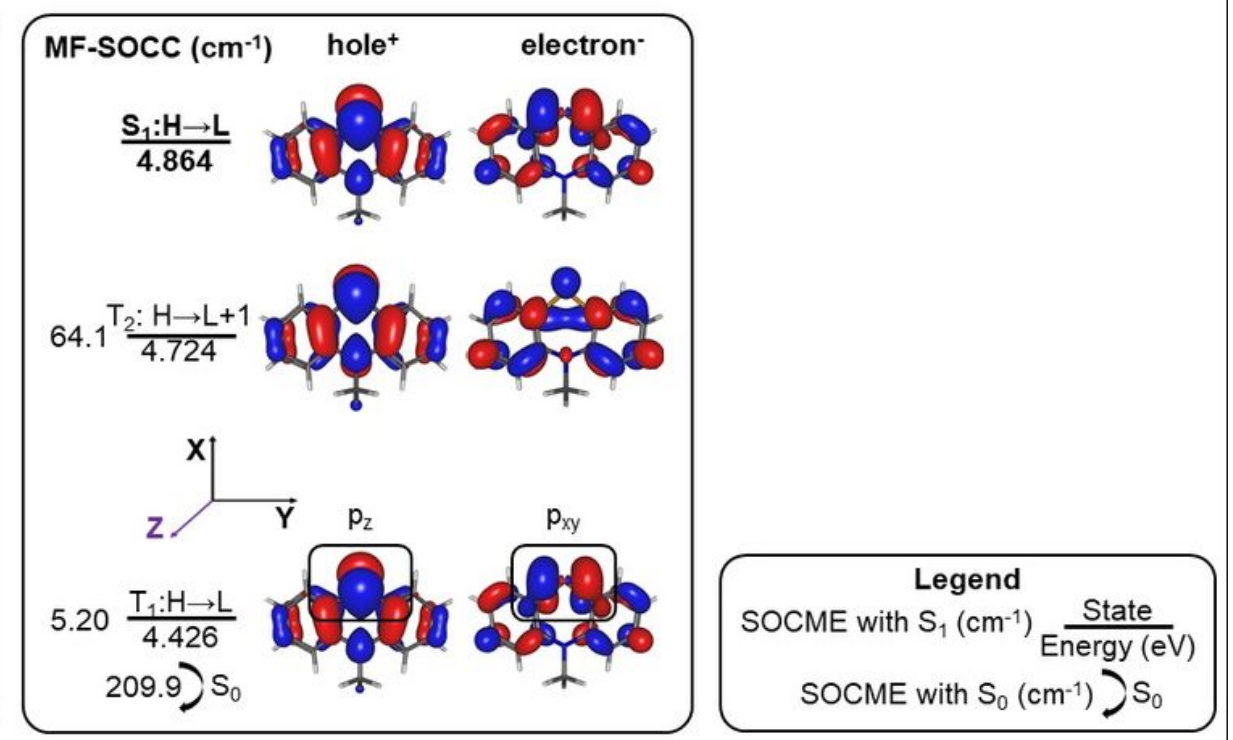

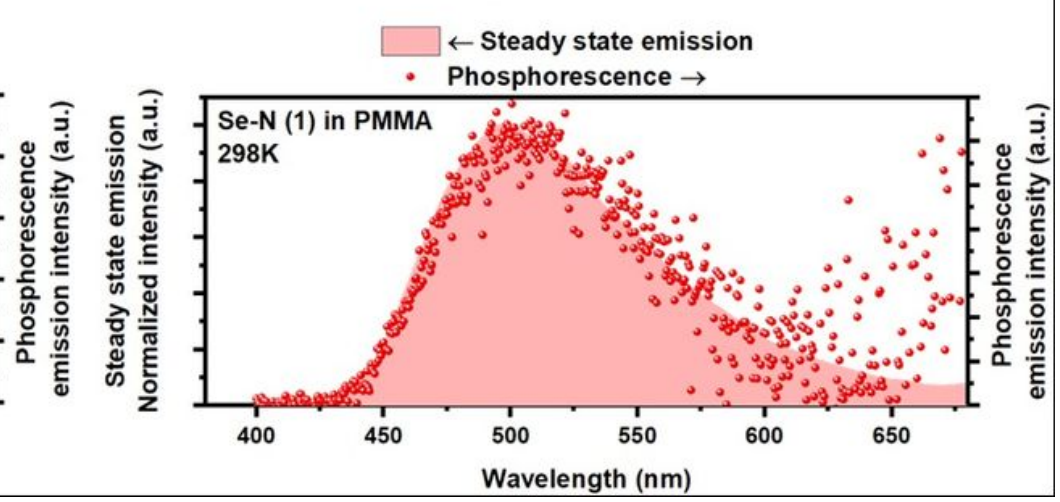

Figure 2

Computational and experimental results of Se-N (1) and Se-N (2). (a) Chemical structures of S N and Se $\mathrm{N}$. Electronic structure results from RAS-SF showing the ground state optimized structure with bent geometry and dihedral angle and the NTOs of S1, T1, T2 states. Energy of each state and the selected mean field SOCMEs are shown as well. (b) Steady state emission spectra (filled graph, left axis) and gated phosphorescence emission spectra (dot, right axis, 0.5-5 ms for Se-N and 0.5-20 ms for S-N) of the two emitters doped in atactic PMMA (1 wt\%, spin-cast) measured at room temperature in vacuum. 

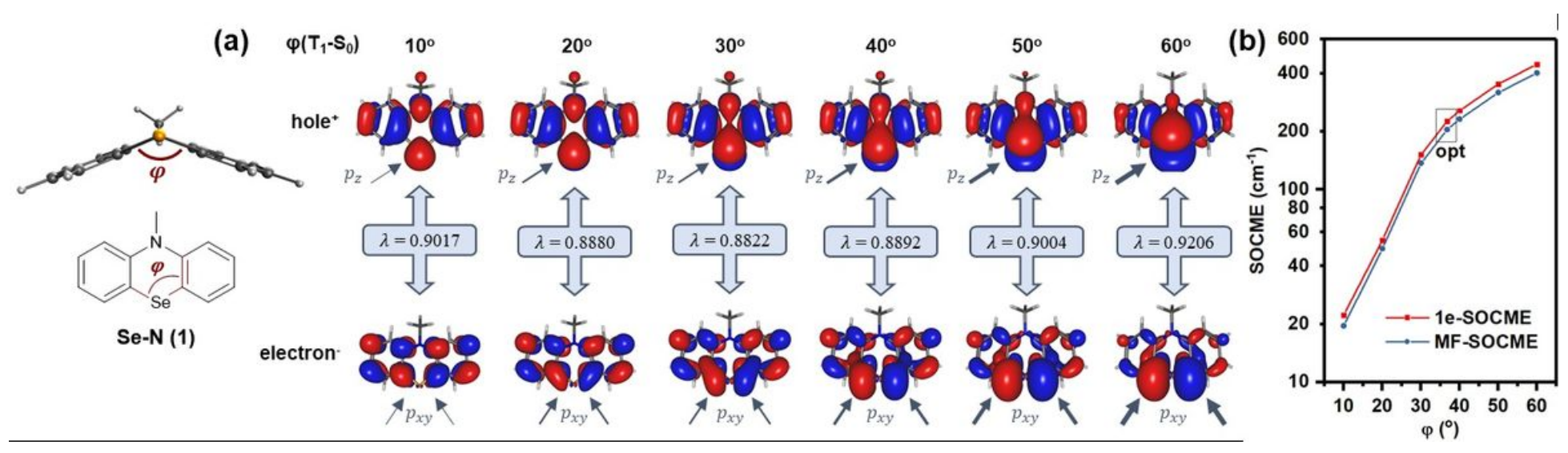

Figure 3

Computational results of Se-N (1) scanned through the dihedral angle. (a) T1-S0 NTOs of Se-N optimized with dihedral angle $(\varphi)$ fixed; $\lambda$ represents the contribution of the NTO pair in SOC (max. 1). (b) RAS SF one-electron and mean-field SOCMEs of T1-S0 transition v.s. dihedral angle, $\varphi(0)$. 
(a)

(X: S or Se)

S-O (3) \& Se-O (4)

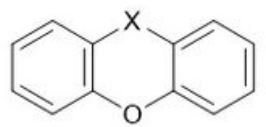

Optimized geometry

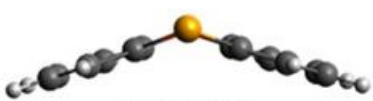

$\varphi: 29.94^{\circ}$

Hole (+)

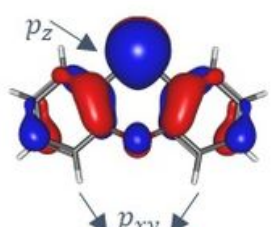

Electron (-)

(b)

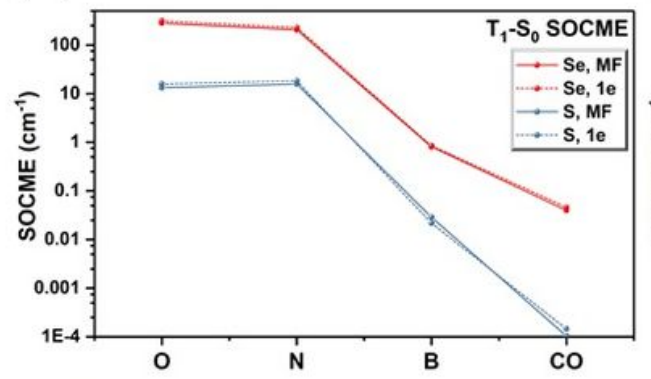

(e)

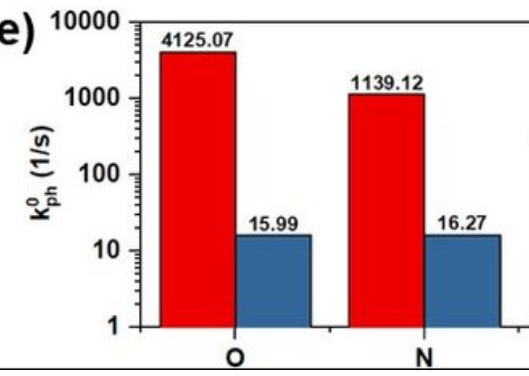

S-N (1) \& Se-N (2)
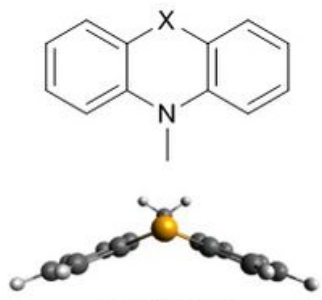

$\varphi: 37.34^{\circ}$
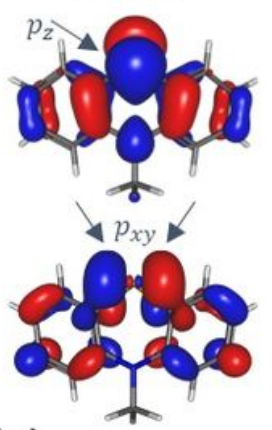

(c)

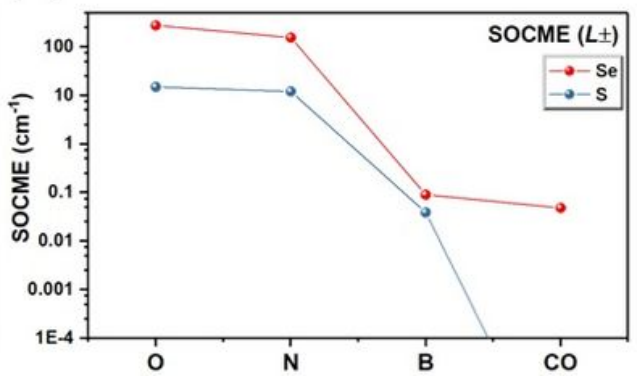

(d)

$\varphi: 8.13^{\circ}$

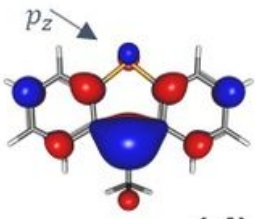

S-CO (7) \& Se-CO (8)
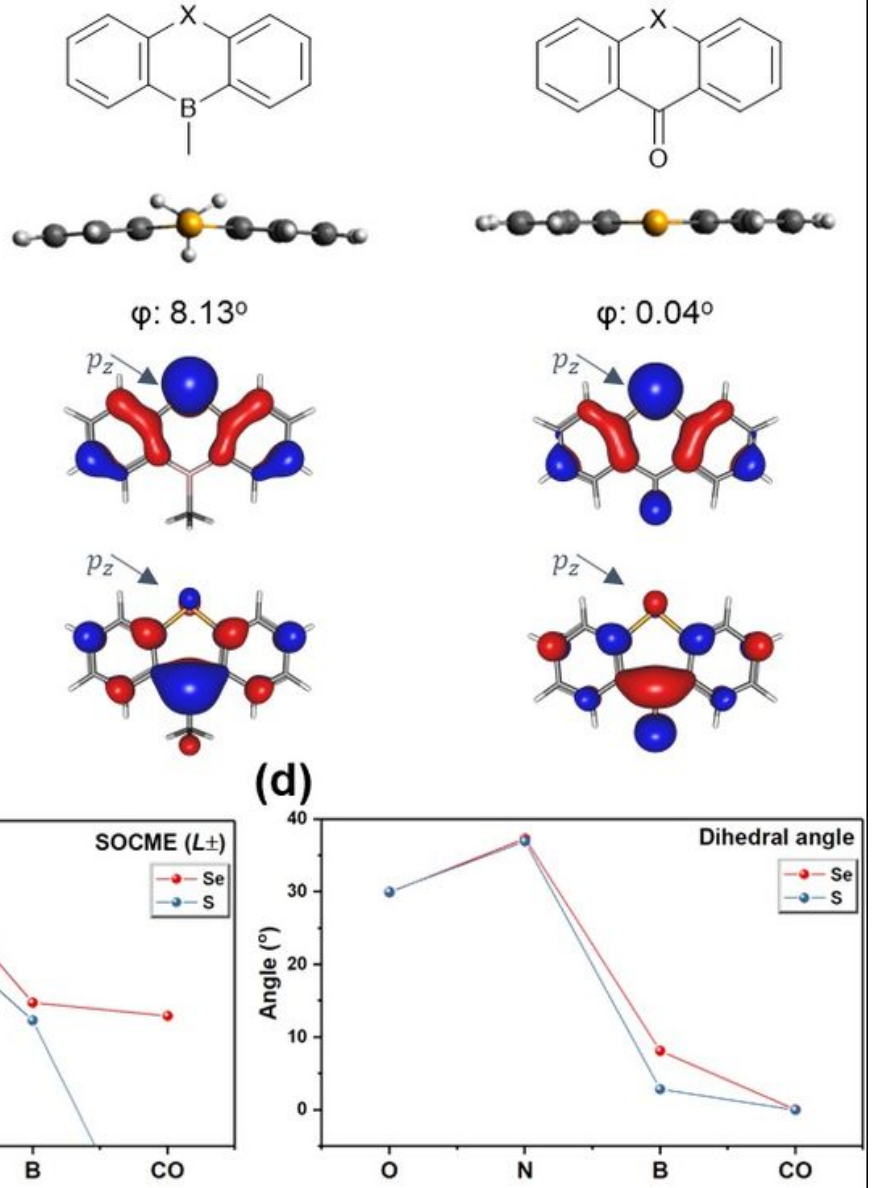

(f)

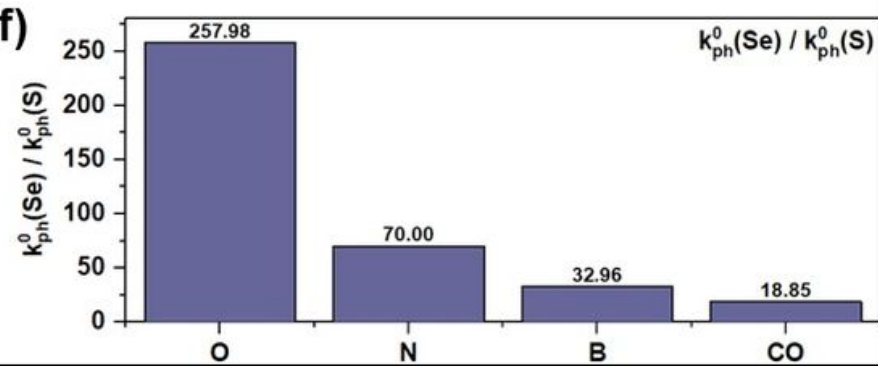

Figure 4

Computational and experimental results for S/Se-O, S/Se-N, S/Se-B, and S/Se-CO. (a) Molecular structures of the oxygen, nitrogen, boron, and carbonyl derivatives $(X=S$ or $S e)$, their optimized ground state geometry with dihedral angle marked, and RAS-SF NTOs of T1 states with Se p_z and p_xy orbitals marked; (b) RAS SF one-electron (1e) and mean-field (MF) SOCMEs of T1-S0 transition, (c) the reduced 1e SOCME in $\mathrm{L}_{-}-$or $\mathrm{L}_{-}+$orientations (modulus) v.s. functional groups; (d) the dihedral angles of molecules studied v.s. their functional groups; (e) the experimental intrinsic phosphorescence rate $k \_p h^{\wedge} 0$ measured in doped PMMA matrix at $78 \mathrm{~K}$ in vacuum, and (f) the $\mathrm{k}_{-} \mathrm{ph}^{\wedge} 0$ (Se derivative)/k_ph^${ }^{\wedge} 0$ (S derivative) value of each functional group.

\section{Supplementary Files}


This is a list of supplementary files associated with this preprint. Click to download.

- SupportingInformationNatChem.pdf 